\title{
Efecto de 2 y 4 meses de práctica de fútbol en el desarrollo osteogénico de niños prepuberales
}

Effect of 2 and 4 months of football practice on the osteogenic development of pre-pubertal children

\author{
Antonio Hernandez-Martin ${ }^{\top}$ * \\ Javier Sanchez-Sanchez ${ }^{2}$ \\ Samuel Manzano-Carrasco \\ Jose Luis Felipe ${ }^{2}$ \\ Leonor Gallardo \\ Jorge García-Unanue \\ 1. IGOID Research Group, Physical Activity and Sport Sciences Department, University of Castilla-La Mancha, 45004 Toledo, Spain. \\ 2. School of Sport Sciences, Universidad Europea de Madrid, 28670 Madrid, Spain.
}

\begin{abstract}
Resumen
El fútbol en edad infantil o juvenil se ha utilizado como una herramienta para mejorar la salud y prevenir futuras enfermedades. El objetivo de este estudio fue investigar el efecto de la participación de 2 y 4 meses en un deporte osteogénico como el fútbol en las variables de contenido mineral óseo y densidad mineral ósea en niños prepuberales. Se incluyeron 20 niños de categoría sub-10 ( $9,5 \pm$ 1,4 años) que realizaban entrenamiento de fútbol 3 horas a la semana y se encontraban en la etapa de Tanner I. Los valores de densidad mineral ósea y contenido mineral óseo se midieron mediante un absorciómetro de rayos $X$ de doble energía. Los resultados mostraron un incremento de los valores de contenido mineral óseo y densidad mineral ósea total tras los dos meses de práctica ( $p<0,05 ;$ TE: 0,11 y TE: 0,27 respectivamente), valores significativamente mayores tras cuatro meses de práctica en comparación con el valor inicial y el valor tras dos meses ( $p<0,05 ;$ TE: 0,40 y TE: 0,13 respectivamente). Aumento significativo de contenido mineral óseo y densidad mineral ósea en las piernas a los dos y cuatro meses de práctica ( $p<0,05 ;$ TE: 0,43 y TE: 0,40 respectivamente). Los datos sugieren que un programa de entrenamiento de fútbol recreativo de 2 y 4 meses es beneficioso para el desarrollo osteogénico y la salud de los niños durante la etapa prepuberal.
\end{abstract}

Palabras clave: Fútbol; niños; prepuberales; contenido mineral óseo; densidad mineral ósea

\begin{abstract}
Football for children and young people has been used as a tool to improve health and prevent future diseases. The aim of this study was to investigate, the effect of 2- and 4-month participation in an osteogenic sport such as football on the variables of bone mineral content and bone mineral density in pre-pubertal children. Twenty under-10 children ( $9.5 \pm 1.4$ years old $)$ who were doing football training 3 hours a week and were at the Tanner I stage were included. Bone mineral density and bone mineral content values were measured using a dual energy X-ray absorber. The results showed an increase in the values of bone mineral content and total bone mineral density after two months of practice ( $p<0.05$; TE: 0.11 and TE: 0.27 respectively), significantly higher values after four months of practice compared to the initial value and the value after two months ( $p<0.05 ;$ TE: 0.40 and TE: 0.13 respectively). Significant increase in bone mineral content and bone mineral density in the legs after two and four months of practice ( $p<0.05 ;$ TE: 0.43 and TE: 0.40 respectively). Our data suggest that a 2- and 4-month recreational football training programme is beneficial for the osteogenic development and health of children during the pre-pubertal stage.
\end{abstract}

Keywords: Football; children; pre-pubescent; bone mineral content; bone mineral density.

*Autor de correspondencia: Antonio Hernandez-Martin; Antonio.HMartinSan@uclm.es

Recibido: 14 de diciembre de 2020

Aceptado: 01 de julio de 2021

Publicado: 01 de diciembre de 2021

Como citar (APA): Hernandez-Martin, A., Sanchez-Sanchez, J., Manzano-Carrasco, S., Luis Felipe, J., Gallardo, L., \& García-Unanue, J. (2021). Efecto de 2 y 4 meses de práctica de fútbol en el desarrollo osteogénico de niños prepuberales. JUMP, (4), 26-32. https://doi.org/10.17561/jump.n4.3 


\section{Introducción}

A largo plazo, la inactividad física se relaciona con un estilo de vida sedentario, que ha sido asociado a enfermedades con elevadas repercusiones económicas vinculadas con el tratamiento y la rehabilitación en diferentes poblaciones, tales como la osteoporosis (Cruz et al., 2009). Esta afección osteoarticular se caracteriza por una baja densidad ósea y es muy común en mujeres y ancianos (Bliuc, Alarkawi, Nguyen, Eisman \& Center, 2015). Multitud de factores influyen en la aparición y desarrollo de osteoporosis, pero una adquisición de masa ósea en la infancia disminuye el riesgo de osteoporosis en la etapa adulta (Jarrosay, Speck, Fernandez, Duvergel \& Martínez, 2016; Hasselstrøm et al., 2007).

Una herramienta para mejorar y aumentar el desarrollo óseo, y por lo tanto, de disminuir el riesgo de padecer osteoporosis es la actividad física (Bartra et al., 2019). En la etapa infantil, un incremento de la actividad física produciría un almacenamiento de masa ósea y con ello, una reducción del riesgo de sufrir fracturas óseas en edad adulta y tercera edad (Karlsson, Nordqvist, \& Karlsson, 2008). Este aumento se produciría siguiendo las recomendaciones internacionales de al menos 60 minutos o más de actividad física moderada a vigorosa por día y que realicen actividades de fortalecimiento muscular y óseo al menos 3 días a la semana (World Health Organization [WHO], 2010).

Numerosos estudios han investigado el efecto de la actividad física sobre la densidad mineral ósea (DMO) en poblaciones de adultos y ancianos (Gómez-Cabello, Ara, GonzálezAgüero, Casajús \& Vicente-Rodríguez, 2012; Verschueren et al., 2013), sin embargo, existen pocos estudios que hayan analizado el efecto del ejercicio en el crecimiento óseo de los niños y su relación con la prevención de las enfermedades óseas en la edad adulta. Asimismo, según Bailey, Martin, McKay, Whiting y Mirwald (2000), un alto porcentaje del contenido mineral óseo (BMC) se logra en torno a los 12 años en los niños, aumentando este efecto con el ejercicio físico antes de la pubertad. Más del $50 \%$ de los casos de osteoporosis en edad adulta, se relacionan con bajos niveles de BMC durante la edad juvenil (Baroncelli, Bertelloni, Sodini, \& Saggese, 2005). Por estos motivos, observamos que la infancia es clave para obtener un pico máximo de masa ósea y así reducir el riesgo de padecer osteoporosis (Rizzoli, Bianchi, Garabédian, McKay \& Moreno, 2010).

El fútbol es uno de los deportes más practicados en el mundo y su práctica se ha sugerido como una estrategia eficaz para reducir y prevenir enfermedades en la edad adulta (Krustrup et al., 2010). La participación en el fútbol puede aumentar el BMC en las zonas corporales que soportan los impactos (Zouch et al., 2014; Maïmoun et al., 2013). El fútbol implica actividades de alto impacto con potencial para estimular el sistema músculo-esquelético. En este sentido, las investigaciones y los exámenes realizados en los deportes de alto impacto han demostrado los mejores niveles de contenido mineral óseo en los jugadores de fútbol (Ubago-Guisado et al., 2019; Lozano-Berges et al., 2018), ya que el fútbol combina acciones de alta intensidad (es decir, saltos, golpeos, sprints, placajes, cambios de dirección y aceleraciones) intercaladas con fases de recuperación de baja intensidad (Barbero-Álvarez, Coutts, Granda, Barbero-Álvarez \& Castagna, 2009; Bloomfield, Polman \& O'Donoghue, 2007), que influyen positivamente en el desarrollo osteogénico. La mayoría de los estudios han utilizado la absorciometría de rayos $X$ de doble energía (DXA) para evaluar el BMC, la BMD de la zona y el área ósea (Behringer, Gruetzner, McCourt \& Mester, 2014) debido a su bajo costo, radiación y disponibilidad.

Por lo tanto, el propósito de este estudio fue investigar mediante un absorciómetro de rayos $X$ de doble energía (DEXA), el efecto de la participación en un deporte osteogénico como el fútbol durante 2 y 4 meses sobre las variables de BMC y BMD en niños prepuberales.

\section{Método}

\section{Participantes}

La muestra del estudio estuvo compuesta por 20 niños varones sub-10 (9,5 \pm 1,4 años) que practicaban fútbol en un equipo masculino de Toledo (Castilla-La Mancha, España), con una frecuencia de entrenamiento de 3 horas a la semana. Los criterios de inclusión fueron que se encontraran en la etapa de Tanner I (prepuberal), en base a la evaluación de la madurez, y que no sufrieran ninguna lesión o enfermedad que les 
impidiera realizar las pruebas. La tabla 1 muestra los valores de altura, peso e IMC de la muestra.

Tabla 1. Valores de altura, peso e IMC en basal, a los 2 meses y 4 meses.

\begin{tabular}{|c|c|c|c|c|c|c|c|c|c|}
\hline \multirow[b]{2}{*}{$\begin{array}{l}\text { ALTURA } \\
\text { (cm) }\end{array}$} & \multicolumn{3}{|c|}{ Basal } & \multicolumn{3}{|c|}{2 meses } & \multicolumn{3}{|c|}{4 meses } \\
\hline & 136,15 & \pm & 8,78 & 137,23 & \pm & 8,54 & 138,80 & \pm & 8,72 \\
\hline $\begin{array}{l}\text { PESO } \\
\text { (kg) }\end{array}$ & 33,43 & \pm & 5,37 & 34,23 & \pm & 5,52 & 34,78 & \pm & 5,42 \\
\hline $\begin{array}{l}\text { IMC } \\
\left(\mathrm{Kg} / \mathrm{m}^{2}\right)\end{array}$ & 17,98 & \pm & 2,08 & 18,12 & \pm & 2,23 & 18,00 & \pm & 2,03 \\
\hline
\end{tabular}

IMC: Índice de Masa Corporal.

La evaluación de la madurez es necesaria para los estudios que se realizan con niños en etapa de crecimiento, ya que el rango de maduración entre individuos de la misma edad cronológica es amplio, especialmente durante los años de la pubertad (Mirwald, Baxter-Jones, Bailey \& Beunen, 2002). El estado de la pubertad se determinó mediante una autoevaluación basada en diferentes fotografías de las etapas de Tanner (Duke, Litt \& Gross, 1980). El estado de la pubertad para esta investigación se clasificó como prepuberal (etapa I) en todos los casos.

El estudio fue aprobado por el Comité de Ética del Complejo Hospitalario de Toledo (España) y se llevó a cabo en base a los requisitos acordados en la declaración de Helsinki. Todos los niños y sus representantes legales completaron formularios de consentimiento informado por escrito previamente al desarrollo de la investigación.

\section{Diseño del estudio}

Se utilizó un diseño cuasiexperimental. El equipo completó un programa de intervención de fútbol de dos meses, que se desarrollaba en dos sesiones de 60 minutos a la semana, además de un partido de dos partes de 25 minutos durante el fin de semana. Cada sesión se extendía desde las 17:00 hasta las 18:00 horas, consistiendo en un calentamiento (10$15 \mathrm{~min}$ ), ejercicios físicos, técnicos y tácticos destinados a la práctica del fútbol (35-40 min) y un enfriamiento o vuelta a la calma con estiramientos (10 min). El entrenamiento fue dirigido en todo momento por el entrenador del equipo. Todos los niños inscritos completaron el período de intervención.

\section{Procedimiento}

En la segunda semana de febrero, las evaluaciones iniciales o de referencia (previas a la intervención) se llevaron a cabo en condiciones similares a las de las pruebas de familiarización. Las evaluaciones finales (posteriores) tuvieron lugar ocho y dieciséis semanas después de la evaluación previa. Todas las valoraciones se llevaron a cabo a la misma hora, en el mismo orden y con idénticas condiciones de temperatura y descanso que la primera evaluación. La composición corporal fue evaluada tanto en la valoración previa como en las evaluaciones posteriores.

\section{Antropometría básica}

La masa corporal $(\mathrm{kg})$ y la altura $(\mathrm{cm})$ se midieron con la balanza electrónica y el medidor de altura SECA (modelo 711; Seca \& Co, KG, Hamburgo, Alemania; precisión 100 g y 0,1 cm; rango $0-220 \mathrm{~kg}$ y $85-200 \mathrm{~cm}$ ), sin zapatos y sólo en ropa interior. La altura se midió de pie con los talones, las nalgas, la espalda y la región occipital en contacto con la varilla de altura.

\section{Densitometría ósea}

Tanto la masa ósea (BMC en g, y BMD en g/ $\mathrm{cm}^{2}$ ) como la masa grasa (\%) fueron calculadas usando un Densitómetro Óseo (Hologic Series Discovery QDR, Software Physician's Viewer, APEX System Software Version 3.1.2. Bedford, MA, USA). El densitómetro óseo se calibró antes de cada día de prueba utilizando un fantoma (estándar de referencia con densidad conocida que simula los puntos anatómicos) medidos de columna lumbar y siguiendo las directrices establecidas por el fabricante. Los participantes fueron evaluados en posición supina, con el cuerpo y las extremidades completamente extendidos, y dentro de los límites establecidos por las líneas de exploración de la camilla. Siendo una densitometría de cuerpo entero. Todas las pruebas y los análisis subsiguientes fueron realizados por el mismo examinador para asegurar la consistencia de las medidas (Esfahanizadeh et al., 2013). 


\section{Análisis estadístico}

Los resultados se muestran con la media \pm desviación estándar. En primer lugar, la normalidad de las variables fue comprobada mediante la prueba de Kolmogorov-Smirnov, concluyendo que todas las variables presentaron una distribución normal $(p>0,05)$. A fin de determinar los efectos de la intervención sobre las variables analizadas, se utilizó la prueba ANOVA de medidas repetidas para la comparación intra-grupo en los tres momentos de valoración, así como el post-hoc de Bonferroni para las comparaciones por pares. La altura y la edad fueron utilizadas como covariables. Además, se incluyó el tamaño del efecto (ES; Cohen's d). El ES se evaluó de la siguiente manera: 0-0,2 = trivial, $0,2-0,5=$ pequeño, $0,5-0,8=$ moderado, $y>$ 0,8 alto (Batterham \& Hopkins, 2006). El valor de significación se delimitó en $p<0,05$.

\section{Resultados}

La tabla 2 muestra los resultados, obteniendo un aumento del BMC y BMD total tras los dos meses de práctica ( $p<0,05$; TE: 0,11 y TE: 0,27, respectivamente). Además, el BMD presenta valores significativamente mayores tras cuatro meses de práctica en comparación con el valor inicial y el valor tras dos meses $(p<0,05$; TE: 0,40 y TE: 0,13, respectivamente). En el caso del BMC en el tronco, los participantes mostraron significativamente mayor cantidad de $\mathrm{g}$ tras cuatro meses respecto al valor inicial $(p<0,05 ;$ TE: 0,17$)$.

En el caso de los brazos, el BMC es significativamente mayor tras cuatro meses respecto a los dos meses $(p<0,05$; TE: 0,26$)$ y el BMD es mayor a los dos meses respecto al valor inicial ( $p<0,05 ;$ TE: 0,15$)$.

Los resultados de las piernas son los que muestran una tendencia más clara. Los resultados del BMC y BMD tras dos meses de práctica son significativamente mayores que el valor inicial ( $p<0,05$; TE: 0,12 y TE: 0,20, respectivamente), mientras que los valores tras cuatro meses fueron significativamente mayores en comparación tanto a los iniciales ( $p<0,05$; TE: 0,43 y TE: 0,40, respectivamente) como a los obtenidos tras dos meses $(p<0,05$; TE: 0,26 y TE: 0,20, respectivamente).

\section{Discusión}

Esta investigación tenía por objeto analizar mediante un absorciómetro de rayos $X$ de doble energía (DEXA) el efecto de la participación de 2 y 4 meses en un deporte osteogénico como el fútbol en las variables de BMC y BMD en niños prepuberales. El ejercicio recomendado para mejorar el BMC y el BMD se basa principalmente en el impacto, es decir, ejercicios pliométricos, saltos, carreras y cualquier actividad basada en el propio peso corporal, como el fútbol, el baloncesto y el balonmano (Asikainen et al., 2004). Los resultados más relevantes se encontraron en el BMD total, que presentó mejores niveles tras cuatro meses de práctica en comparación con el valor inicial y respecto al valor tras dos meses de entrenamiento $(p<0,05)$. Al analizar según la zona corporal, destacó que el BMC y BMD en las piernas tras dos meses de práctica fueron significativamente mayores respecto al inicial $(p<0,05)$, mientras que los valores tras cuatro meses fueron significativamente mayores que los iniciales $(p<0,05)$ y que los obtenidos tras dos meses de intervención $(p<0,05)$.

En el presente estudio se analizaron las variables de densidad mineral ósea y contenido mineral óseo de los niños prepúberes en cuerpo entero, tronco, brazos y piernas, incluyendo la masa grasa. En cuanto a la masa ósea, el equipo mostró un aumento de BMC y BMD a lo largo de las dos valoraciones (2 meses y 4 meses) en todo el cuerpo, tronco, brazos y piernas. Esto podría deberse a que un deporte como el fútbol se asocia con ejercicios de alto impacto (Helge et al., 2014; Vicente-Rodriguez, Ara, Pérez-Gómez, Dorado \& Calbet, 2005), ya que implica sprints, saltos y movimientos que requieren cambios bruscos en el centro de masa (Seabra et al., 2012). Los resultados del estudio también podrían explicarse por los ejercicios pliométricos incluidos en el entrenamiento de fútbol, ya que según la literatura previa estos ejercicios presentan un gran potencial para inducir una mayor masa ósea en los niños (Gunter et al., 2008). Estos resultados son similares a los hallados en estudios previos como el de Zouch 
et al. (2008), donde los niños que jugaban al fútbol mostraron niveles más altos de BMC y BMD que los del grupo control. Además, en la presente investigación se encontraron valores significativamente mayores de BMC y BMD en las extremidades inferiores con el paso del tiempo. Este hallazgo es similar a los resultados obtenidos en estudios anteriores como el de Zouch et al. (2008) y va en línea con los datos presentados por VicenteRodríguez et al. (2005), quienes demostraron que los niños que practicaban fútbol sufrían un aumento significativo del BMC en las extremidades inferiores con respecto a las superiores. Sin embargo, Larsen et al. (2017) no encontraron ninguna mejora significativa en cuando a la BMC en las piernas. Las causas de estas diferencias están marcadas por la mayor actividad física que tienen que soportar los huesos de la pierna, ya que están continuamente soportando el cuerpo y las acciones del balón. Por otro lado, siguiendo el estudio de Zouch et al. (2008), el BMC podría deberse al nivel de desarrollo en el que se encuentran los niños, ya que la pubertad es la etapa en la que los niños experimentan una mayor curva de crecimiento (Gomez-Campos et al., 2019). Por otro lado, también se observó una disminución del porcentaje de masa grasa con el entrenamiento. A este respecto, cabe destacar que la práctica del fútbol podría prevenir enfermedades como la obesidad infantil o el sobrepeso (Krustrup et al., 2010). Esta disminución de masa grasa podría haber sido debida al alto impacto de las acciones que tienen lugar durante la práctica del fútbol, que potencian el aumento del tejido muscular y favorecen la disminución de la masa grasa (Helge et al., 2014).
La principal limitación de este estudio fue la falta de un grupo control, así como la cuantificación de la carga externa y la intensidad de los entrenamientos y los partidos. Además, los participantes no jugaron la misma cantidad de minutos en todos los partidos y esto podría haber afectado en cierta media los resultados obtenidos. En futuras investigaciones, podría ser interesante desarrollar este estudio longitudinalmente para ver si existe una relación causa-efecto, con niños que no hayan realizado previamente fútbol.

En conclusión, los resultados encontrados en este estudio sugieren que la práctica del fútbol genera beneficios para la salud osteogénica en los niños prepúberes. Especialmente en los niveles osteogénicos de las piernas. Estos beneficios aparecen a las 8 semanas de práctica, y son aún mayores a las 16 semanas de práctica.

\section{Aplicaciones prácticas}

Estos resultados, pueden ser muy útiles tanto para los formadores, entrenadores y padres de estos niños, ya que no sólo se divierten realizando deporte, sino que gracias a ello pueden prevenir enfermedades $y$ lesiones en su etapa adulta y tercera edad.

Como perspectiva de futuro, se deberán realizar estudios analizando la carga y demandas físicas que soportan los niños practicando fútbol y su relación con las mejoras osteogénicas. A su vez, un estudio longitudinal de varios años observando el desarrollo total de los niños e identificar el efecto del fútbol en ese desarrollo sería idóneo para seguir avanzando en el conocimiento.

Tabla 2.

\begin{tabular}{lccccccccc}
\hline & \multicolumn{3}{c}{ Basal } & \multicolumn{4}{c}{2 meses } & \multicolumn{3}{c}{4 meses } \\
\hline \% Grasa $(\%)$ & 21,29 & \pm & 6,40 & 21,88 & \pm & $6,58 \mathrm{a}$ & 20,90 & \pm & $6,41 \mathrm{~b}$ \\
CMO Total $(\mathrm{g})$ & 1075,47 & \pm & 151,08 & 1092,73 & \pm & $157,36 \mathrm{a}$ & 1108,34 & \pm & 149,87 \\
DMO Total $\left(\mathrm{g} / \mathrm{cm}^{2}\right)$ & 0,68 & \pm & 0,07 & 0,70 & \pm & $0,08 \mathrm{a}$ & 0,71 & \pm & $0,08 \mathrm{a}, \mathrm{b}$ \\
CMO Tronco $(\mathrm{g})$ & 277,09 & \pm & 47,22 & 280,99 & \pm & 48,80 & 285,45 & \pm & $51,88 \mathrm{a}$ \\
CMO Brazos $(\mathrm{g})$ & 52,69 & \pm & 8,65 & 52,35 & \pm & 8,54 & 54,69 & \pm & $9,17 \mathrm{~b}$ \\
DMO Brazos $\left(\mathrm{g} / \mathrm{cm}^{2}\right)$ & 0,54 & \pm & 0,06 & 0,55 & \pm & $0,07 \mathrm{a}$ & 0,54 & \pm & 0,07 \\
CMO Piernas $(\mathrm{g})$ & 188,00 & \pm & 39,05 & 194,64 & \pm & $40,83 \mathrm{a}$ & 205,98 & \pm & $45,14 \mathrm{a}, \mathrm{b}$ \\
DMO Piernas $\left(\mathrm{g} / \mathrm{cm}^{2}\right)$ & 0,79 & \pm & 0,10 & 0,81 & \pm & $0,10 \mathrm{a}$ & 0,83 & \pm & $0,10 \mathrm{a}, \mathrm{b}$ \\
\hline
\end{tabular}

Valores de composición corporal 


\section{Referencias}

Asikainen, T.-M., Kukkonen-Harjula, K., \& Miilunpalo, S. (2004). Exercise for health for early postmenopausal women: A systematic review of randomised controlled trials. Sports Medicine, 34(11), 753-778. https://doi.org/10.2165/00007256-200434110-00004

Bailey, D. A., Martin, A. D., McKay, H. A., Whiting, S., \& Mirwald, R. (2000). Calcium accretion in girls and boys during puberty: A longitudinal analysis. Journal of Bone and Mineral Research, 15(11), 2245-2250. https://doi.org/10.1359/jbmr.2000.15.11.2245

Barbero-Álvarez, J. C., Coutts, A., Granda, J., Barbero-Álvarez, V., \& Castagna, C. (2009). The validity and reliability of a global positioning satellite system device to assess speed and repeated sprint ability (RSA) in athletes. Journal of Science and Medicine in Sport, 13(2), 232-235. https://doi.org/10.1016/j.jsams.2009.02.005

Baroncelli, G. I., Bertelloni, S., Sodini, F., \& Saggese, G. (2005). Osteoporosis in children and adolescents. Pediatric Drugs, 7(5), 295-323. https://doi.org/10.2165/00148581-200507050-00003

Bartra, A., Caeiro, J. R., Mesa-Ramos, M., Etxebarría-Foronda, I., Montejo, J., Carpintero, P., ... \& Canals, L. (2019). Cost of osteoporotic hip fracture in Spain per Autonomous Region. Revista Española de Cirugía Ortopédica y Traumatología (English Edition), 63(1), 56-68. https://doi.org/10.1016/j.recote.2018.11.004

Batterham, A. M., \& Hopkins, W. G. (2006). Making meaningful inferences about magnitudes. International Journal of Sports Physiology and Performance, 1, 50- 57. https://doi.org/10.1123/ijspp.1.1.50

Behringer, M., Gruetzner, S., McCourt M., \& Mester, J. (2014). Effects of weight-bearing activities on bone mineral content and density in children and adolescents: a meta-analysis. Journal Bone Mineral Research, 29(2), 467-478. https://doi.org/10.1002/jbmr.2036

Bliuc, D., Alarkawi, D., Nguyen, T. V., Eisman, J. A., \& Center, J. R. (2015). Risk of subsequent fractures and mortality in elderly women and men with fragility fractures with and without osteoporotic bone density: the Dubbo Osteoporosis Epidemiology Study. Journal of bone and mineral research, 30(4), 637-646. https://doi.org/10.1002/jbmr.2393

Bloomfield, J., Polman, R., \& O'Donoghue, P. (2007). Physical demands of different positions in FA Premier League soccer. Journal of Sports Science and Medicine, 6(1), 63-70. https://www.ncbi.nlm.nih.gov/pmc/articles/PMC3778701/

Cruz, J. G., Martínez, R. F., Martínez, J. G., Gutiérrez, E. S., Serrano, M. E., \& De Las Deses, C. D. H. (2009). Osteoporosis. Conceptos básicos para la práctica diaria. Revista de Especialidades Médico-Quirúrgicas, 14(3), 128-140.

https://www.medigraphic.com/pdfs/quirurgicas/rmq-2009/ rmq093f.pdf

Duke, P. M., Litt, I. F., \& Gross, R. T. (1980). Adolescents' selfassessment of sexual maturation. Pediatrics, 66(6), 918-920.

Esfahanizadeh, N., Davaie, S., Rokn, A. R., Daneshparvar, H. R., Bayat, N., Khondi, N., ... \& Ghandi, M. (2013). Correlation between bone mineral density of jaws and skeletal sites in an Iranian population using dual X-ray energy absorptiometry. Dental research journal, 10(4), 460. http://www.ncbi.nlm.nih.gov/pmc/articles/pmc3793408/

Gómez-Cabello, A., Ara, I., González-Agüero, A., Casajús, J. A., \& Vicente-Rodríguez, G. (2012). Effects of training on bone mass in older adults: A systematic review. Sports Medicine, 42(4), 301-325. https://doi.org/10.2165/11597670-000000000-00000

Gomez-Campos, R., Santi-Maria, T., Arruda, M., Maldonado, T., Albernaz, A., Schiavo, M., \& Cossio-Bolaños, M. (2019). Fatfree mass and bone mineral density of young soccer players: proposal of equations based on anthropometric variables. Frontiers in psychology, 10, 522. https://doi.org/10.3389/fpsyg.2019.00522

Gunter, K., Baxter-Jones, A. D., Mirwald, R. L., Almstedt, H., Fuchs, R. K., Durski, S., \& Snow, C. (2008). Impact exercise increases BMC during growth: an 8-year longitudinal study. Journal of Bone and Mineral Research, 23(7), 986-993. https://doi.org/10.1016/j.bone.2008.01.002

Hasselstrøm, H., Karlsson, K. M., Hansen, S. E., Grønfeldt, V., Froberg, K., \& Andersen, L. B. (2007). Peripheral bone mineral density and different intensities of physical activity in children 6-8 years old: the Copenhagen School Child Intervention study. Calcified tissue international, 80(1), 31-38. https://doi.org/10.1007/s00223-006-0137-9

Helge, E. W., Andersen, T. R., Schmidt, J. F., Jørgensen, N. R, Hornstrup, T., Krustrup, P., \& Bangsbo, J. (2014). Recreational football improves bone mineral density and bone turnover marker profile in elderly men. Scandinavian journal of medicine \& science in sports, 24(S1), 98-104. https://doi.org/10.1111/sms.12239

Jarrosay, L. F., Speck, C. M. J., Fernandez, G. S., Duvergel, N. S., \& Martínez, R. H. (2016). Osteoporosis. Problema social actual. Revista Información Científica, 95(6), 1052-1066.

Karlsson, M., Nordqvist, A., \& Karlsson, C. (2008). Physical activity increases bone mass during growth. Food \& nutrition research 52(1), 1871. https://doi.org/10.1139/y96-099

Krustrup, P., Aagaard, P., Nybo, L., Petersen, J., Mohr, M., \& Bangsbo, J. (2010). Recreational football as a health promoting activity: A topical review. Scandinavian Journal of Medicine \& Science in Sports, 20(s1), 1-13. https://doi.org/10.1111/j.1600-0838.2010.01108.x

Larsen, M. N., Nielsen, C. M., Ørntoft, C., Randers, M. B., Helge, E. W., Madsen, M., ... \& Krustrup, P. (2017). Fitness effects of 10 -month frequent low-volume ball game training or interval running for 8-10-year-old school children. BioMed research international. https://doi.org/10.1155/2017/2719752

Lozano-Berges, G., Matute-Llorente, Á., González-Agüero, A., Gómez-Bruton, A., Gómez-Cabello, A., Vicente-Rodriguez, G. \& Casajús, J. A. (2018). Soccer helps build strong bones during growth: a systematic review and meta-analysis. European journal of pediatrics, 177(3), 295-310. https://doi.org/10.1007/s00431-017-3060-3

Maïmoun, L., Coste, O., Philibert, P., Briot, K., Mura, T., Galtier, F., ... \& Sultan, C. (2013). Peripubertal female athletes in high-impact sports show improved bone mass acquisition and bone geometry. Metabolism, 62(8), 1088-1098. https://doi.org/10.1016/j.metabol.2012.11.010

Mirwald, R. L., Baxter-Jones, A. D., Bailey, D. A., \& Beunen, G. P. (2002). An assessment of maturity from anthropometric measurements. Medicine \& science in sports \& exercise, 34(4), 689-694. https://doi.org/10.1097/00005768-200204000-00020

Rizzoli, R., Bianchi, M. L., Garabédian, M., McKay, H. A., \& Moreno, L. A. (2010). Maximizing bone mineral mass gain during growth for the prevention of fractures in the adolescents and the elderly. Bone, 46(2), 294-305. https://doi.org/10.1016/j.bone.2009.10.005

Seabra, A., Marques, E., Brito, J., Krustrup, P., Abreu, S., Oliveira, J., Rebelo, A. (2012). Muscle strength and soccer practice as major determinants of bone mineral density in adolescents. Joint Bone Spine, 79(4), 403-408. https://doi.org/10.1016/j.jbspin.2011.09.003

Ubago-Guisado, E., Vlachopoulos, D., Barker, A. R., Christoffersen, T., Metcalf, B., \& Gracia-Marco, L. (2019). Effect of maturational timing on bone health in male adolescent athletes engaged in different sports: the PRO-BONE study. Journal of science and medicine in sport, 22(3), 253-258. https://doi.org/10.1016/j.jsams.2018.08.009 
Verschueren, S., Gielen, E., O'Neill, T. W., Pye, S. R., Adams, J. E., Ward, K. A., \& Boonen, S. (2013). Sarcopenia and its relationship with bone mineral density in middle-aged and exercise on bone mass development. Journal of Bone and Mineral Metabolism 26, 416-424. https://doi.org/10.1007/s00198-012-2057-z

Vicente-Rodriguez, G., Ara, I., Pérez-Gómez, J., Dorado, C., \& Calbet, J. A. (2005). Muscular development and physical activity as major determinants of femoral bone mass acquisition during growth. British Journal of Sports Medicine, 39(9), 611-616. http://dx.doi.org/10.1136/bjsm.2004.014431
World Health Organization [WHO]. (2010). Medical eligibility criteria for contraceptive use. World Health Organization.

Zouch, M., Jaffré, C., Thomas, T., Frère, D., Courteix, D., Vico, L., \& Alexandre, C. (2008). Long-term soccer practice increases bone mineral content gain in prepubescent boys. Joint Bone Spine, 75(1), 41-4. https://doi.org/10.1016/j.jbspin.2006.12.008

Zouch, M., Vico, L., Frere, D., Tabka, Z., \& Alexandre, C. (2014). Young male soccer players exhibit additional bone mineral acquisition during the peripubertal period: 1-year longitudinal study. European Journal Pediatric, 173(1),53-61. https://doi.org/10.1007/s00431-013-2115-3 